\title{
The Development of in-Situ, Photo Crosslinked, Citric Acid Based Polymeric Delivery Systems
}

\author{
Kiran Dhaliwal* and P Dosanjh \\ UCL Centre for Nanotechnology and Regenerative Medicine, Royal Free London NHS Foundation Trust, Pond Street, London NW3 2QG, United Kingdom
}

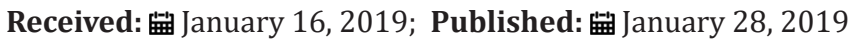

*Corresponding author: Kiran Dhaliwal, UCL Centre for Nanotechnology and Regenerative Medicine, Royal Free London NHS

Foundation Trust, Pond Street, London NW3 2QG, United Kingdom

\begin{abstract}
Background: Biodegradable polymers are currently limited in their application in engineering of soft tissues because they are too stiff and incompliant. Biodegradable elastomers overcome these limitations; they have ability to recover from multiple deformations. Citric acid based biodegradable polymers (CABEs) are a family of elastomers that have been recently developed. Poly octamethylene maleate citrate (POMC) is a promising novel co- polymer with several potential applications including drug delivery. However, further research is required to understand its full potential. There is also a need for more gentile and facile methods of biological molecule inclusion. Photo-polymerisation provides an effective solution. In this study the inclusion of biologically active molecules (Dexamethasone and Bovine Serum Albumin) are included in a POMC delivery construct, using photopolymerization techniques and the subsequent release is reported.
\end{abstract}

Method: POMC is formed via a poly-condensation reaction. Two different compositions of pre-POMC were synthesised (6:4 and 8:2 ratios) Both UV and blue light systems were used to crosslink the pre-POMC polymer. The pre-POMC and crosslinked POMC polymer were characterised using FTIR spectroscopy. The crosslinked POMC was thermally analysed via DSC to determine the glass transition temperature. Tensile strength was determined via tensile testing. Drug/protein release was measured using a spectrophotometer. The degradation rate was investigated by measuring mass loss of the POMC disks placed in PBS solution.

Results: The results indicate that that both 6:4 and 8:2 pre-POMC can be successfully synthesised. However, only 6:4 POMC was successfully crosslinked using both UV and blue light. Photo-polymerisation was used to crosslink the 6:4 pre-polymer and include drug and protein molecules. The study also demonstrates successful controlled and sustained release of Dexamethasone and BSA over a 14-day period.

Conclusion: This study demonstrates that pre-POMC can be successfully synthesised and crosslinked to form POMC using both UV and blue light systems. Furthermore, this system can be used to successfully incorporate biologically active molecules into the polymer and has demonstrated successful sustained release. The results of this study suggest that this is a very versatile system that can potentially be successfully used in a variety of different applications.

Abbreviations: CABEs: Citric Acid Based Biodegradable Polymers; PMOC: Poly Octamethylene Maleate Citrate; PHAs: Polyhydroxyalkanoates; HASMC: Human Aortic Smooth Cells; HAEC: Human Aortic Endothelial Cells; POC: Poly Octanediol-Co-Citrate; DMSO: Dimethylsulfoxide; BSA: Bovine Serum Albumin; PBS: Phosphate Buffer Solution; FTIR: Fourier Transform Infra-Red; DSC: Differential Scanning Calorimetry

\section{Introduction}

Citric acid based biodegradable polymers CABEs are a family of polyester elastomers that have been recently developed. Poly (diol citrates) were the first group of CABEs to be synthesised and have been most widely studied. Synthesis of poly (diol citrates) is based on polymerisation of a linear diol with citric acid. Citric acid is a multifunctional molecule which provides pendant functionality and is a non-toxic by-product of the Krebs cycle. It is FDA approved and currently used in clinical settings as an anticoagulant [1]. The primary advantage of CABEs over other polymers is their ease of synthesis. They are formed via poly-condensation reactions without the use of toxic catalysts or crosslinking reagents [2].
The monomers are also readily available, relatively inexpensive and importantly non-toxic, even once they have been degraded. They are also synthesised in very mild conditions in a short space of time. CABEs can be synthesised at temperatures as low as $1350 \mathrm{C}$ in a reaction taking only 125 minutes, whereas many of the biodegradable polymers reported in the literature have very complex and costly synthesis procedures. For example, the synthesis of polyhydroxyalkanoates (PHAs) [3] has a very complicated fermentation synthesis procedure and the reactions to form polyesters such as PGLA can take up to six hours and requires temperatures of up to $200 \mathrm{oC}$ (Kiremitici-gumusderelioglu and Deniz, 1999). 
Furthermore, the formation of elastomers from polymers of D, L-lactide [4] and the synthesis reaction for PGS can both take up to three days to complete [2]. The poly-condensation reaction produces a crosslinked polyester network with degradable ester bonds [5] and the elastic nature of the molecule is conferred by this crosslinked network. The reaction preserves the pendant functionality of the citric acid molecule for potential conjugation with biological molecules without the need for further modification. This is because citric acid is a multifunctional molecule; it removes the need for further modification of the polymer, saving time and money. The low cost of the monomers and the simple synthesis procedure of CABEs increases their potential for commercialisation. This is an important consideration that can affect the clinical implementation of materials. One of the most unique aspects of CABEs is the ease with which their physical properties can be controlled. These properties have been shown to be controllable by

a) changing the diol used [2]

b) changing the molar ratio of the monomers [6],

c) changing the synthesis conditions, including temperature [6] and

d) changing the polymerisation conditions [8].

Modification of these conditions can also modulate degradation rates [2]; this allows CABEs to be tailored to specific applications unlike other biodegradable polymers. The first CABE was synthesised by Yang, Citric acid was reacted with a range of diols, including 1,8-Octanediol, to create the novel Poly (1,8-octanediolco-citric acid) (POC) biodegradable elastomer. POC demonstrates good mechanical properties with an ultimate tensile strength as high as 6.1 MPa and Youngs modulus with a range of 0.92-16.4 $\mathrm{MPa}$, making it within the range of many soft tissues in the body.
Yang also demonstrated that POC had excellent biocompatibility; it supports the attachment and proliferation of human aortic smooth cells (HASMC) and human aortic endothelial cells (HAEC). The cells were able to attach to the surface without needing any pretreatment or surface modification. POC also demonstrated an in vivo inflammatory response similar to PLGA, a degradable polymer widely accepted as biocompatible. Owing to their unique properties CABEs have been utilised in a wide variety of applications; the most successful being cardiovascular tissue engineering. Yang, developed a biphasic tubular POC scaffold for use as small diameter blood vessel grafts.

The mechanical properties of the scaffold are very similar to properties of native human arteries and veins. In addition, Kibbe, provides evidence for POC as a non-thrombogenic coating for blood contacting devices Kibbe, demonstrated that when current ePTFE grafts are coated in POC it resulted in improved endothelial cell adhesion and reduced platelet adhesion compared to standard ePTFE grafts. Since their initial development their repertoire has greatly expanded due to further research in the area owing to the promising results initial studies into CABEs have produced. Poly (octamethylene maleate citrate) POMC is a promising novel copolymer with several potential applications including drug delivery, cell delivery and wound healing. It was recently synthesised by Gyawali, (Figure 1). POMC is the first biodegradable elastomer that can be crosslinked in situ via photo-polymerisation; it has been shown to be biocompatible and support cell adhesion and proliferation. There is currently only one study that has reported the use of CABEs in drug delivery. Hoshi, used nonporous poly(1,8octanediol-co-citrate) (POC) to form a drug delivery system to entrap Dextran, an anti-platelet drug. The results showed that POC could release Dextran at a slow, controlled rate and therefore could potentially be used in drug delivery applications.

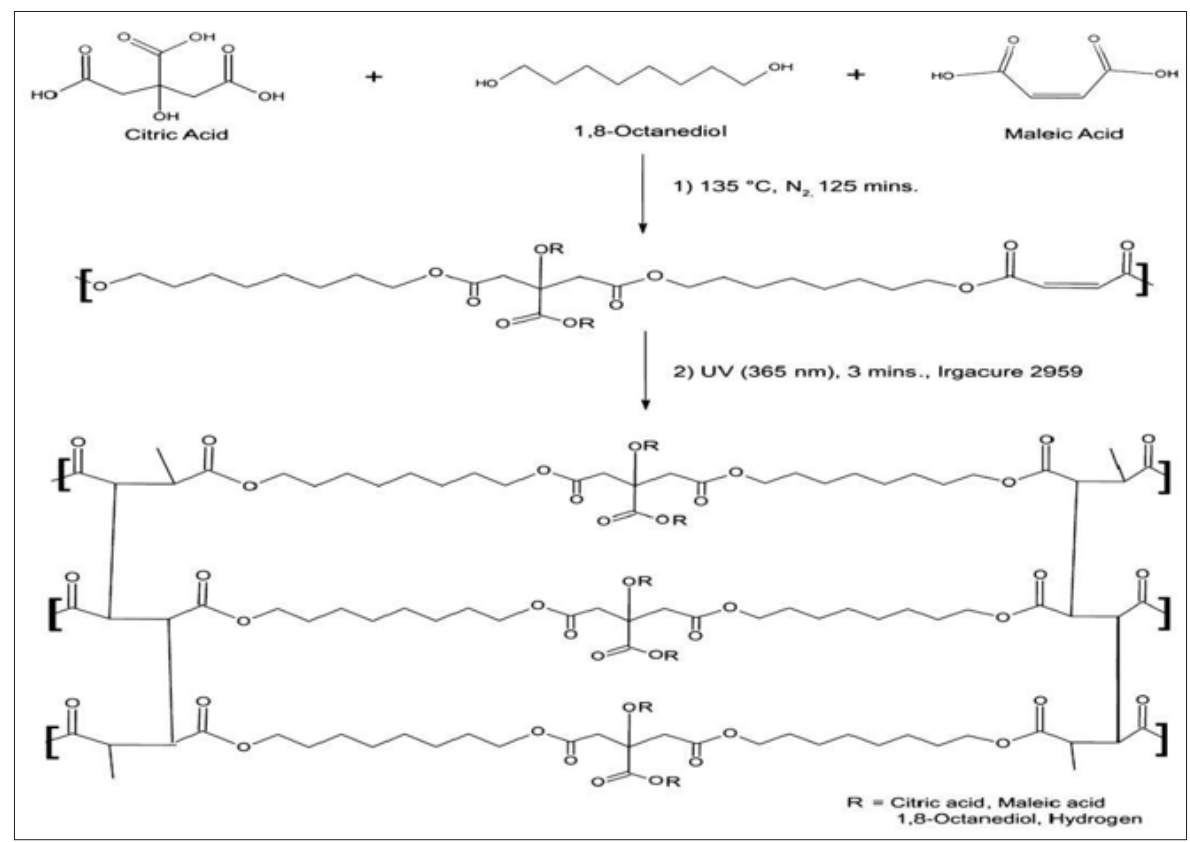

Figure 1: A schematic diagram of the steps involved in POMC synthesis. 
In addition to this, previous studies evaluating the properties of CABEs $[2,6]$ have reported that they have the potential for successful application in drug delivery but have not carried out any controlled release studies. Although in situ polymerisation has its advantages, the conditions required are very specific. The physiologically acceptable temperatures are within a very narrow range and the system must be rapidly polymerised if it is to be successfully implanted in a clinical setting [9]. The advantage of photoinitiated crosslinking is that the reaction proceeds rapidly at low temperatures and therefore may be suitable for the incorporation of thermally sensitive drugs such as peptides and proteins (Amsden, 2008). Photopolymerisable systems have an advantage over other types of in situ systems, such as chemically initiated systems. This was demonstrated by Dunn, who crosslinked biodegradable copolymers D, L lactide and L-lactide with $\varepsilon$-caprolactone, using a chemically initiated thermo-set system, for use as a slow-release drug delivery system. Disadvantages of the system include taking up to 30 minutes to set and the highly exothermic nature of the crosslinking reaction could result in tissue necrosis. There was also burst release of the drug in the first hour which could result in the appearance of side effects.

Drug delivery systems that can be formed in situ have evolved from the need for prolonged and better control of drug administration. They offer a wide range of advantages over other drug delivery systems. Firstly, the drugs can be administered very easily by injecting into the desired site, making it quick and relatively painless for the patient, which can increase patient compliance. They also allow localised and sustained drug release over a prolonged period of time. Furthermore, it can be moulded into any desired shape, regardless of complexity, which is very difficult to achieve with other methods of drug delivery [10]. For example, PGLA microspheres are very difficult to administer because they require a surgical incision to gain access to the desired site. They also require prior preparation before they can be injected into the body, making it inconvenient for the patient [11]. In situ systems are relatively easy to synthesise, making them excellent candidates for clinical use.

\section{Aims}

Although many advances in drug delivery systems and methods have been made over the years, there is still a need for new, unique materials owing to the advances in pharmaceuticals. This study aims to investigate the synthesis of CABEs and subsequently its efficacy as a drug delivery vehicle. Several studies in the literature have reported promising results that suggest the potential use of CABEs in drug delivery systems, however a comprehensive literature review identified only one study to date that actually looks at drug release [12] - there is clearly a distinct lack of research in the area. There is also a need for more gentile and facile methods of biological molecule inclusion. Photo-polymerisation provides an effective solution, yet only two studies to date have demonstrated photo-crosslinking of CABEs [6,7]. This is another area that requires further investigation. Carrying out this study will provide further evidence for the successful application of CABEs in drug delivery as well as photo-polymerisation as a crosslinking technique.
The aims of this study were

a) To synthesise a series of degradable pre-polymers and use photo-polymerisation techniques to crosslink them in order to create novel methods of in situ inclusion of biological molecules.

b) To subsequently use these constructs for protein and drug delivery by incorporating Dexamethasone and BSA into the POMC polymer network and quantify drug and protein release using spectrophotometry.

\section{Methodology \\ POMC Synthesis and Purification}

Pre-POMC was synthesised using citric acid, maleic acid and 1,8 octanediol (Figure 2). After synthesis, pre-POMC was dissolved in 1,4 Dioxane to remove any impurities. Once the pre-POMC had been purified and freeze dried, it was dissolved in Dimethylsulfoxide (DMSO) to form $50 \%(\mathrm{w} / \mathrm{w})$ polymer solution. Dexamethasone and bovine serum albumin (BSA) were incorporated into POMC disks to determine release from POMC. In vitro conditions were simulated using phosphate buffer solution (PBS). PBS solution was made by adding PBS tablets (Oxoid) to distilled water. Each tablet contained sodium chloride $(8.0 \mathrm{~g} / \mathrm{l})$, potassium chloride $(0.2 \mathrm{~g} / \mathrm{l})$, di-sodium hydrogen phosphate $1.15(\mathrm{~g} / \mathrm{l})$ and potassium dihydrogen phosphate $(0.2 \mathrm{~g} / \mathrm{l})$. For every tablet used, $100 \mathrm{ml}$ of distilled water was added. The precipitated polymer was then freeze-dried (Edwards Modulyo K4 freeze dryer) for 24hrs at 600C. Fourier transform infra-red (FTIR) spectroscopy was used to determine the presence of functional groups in pre-POMC. FTIR spectroscopy was carried out at room temperature using an FTIR machine (Perkin Elmer Spectrum One) and run using Spectrum One software [13-21].

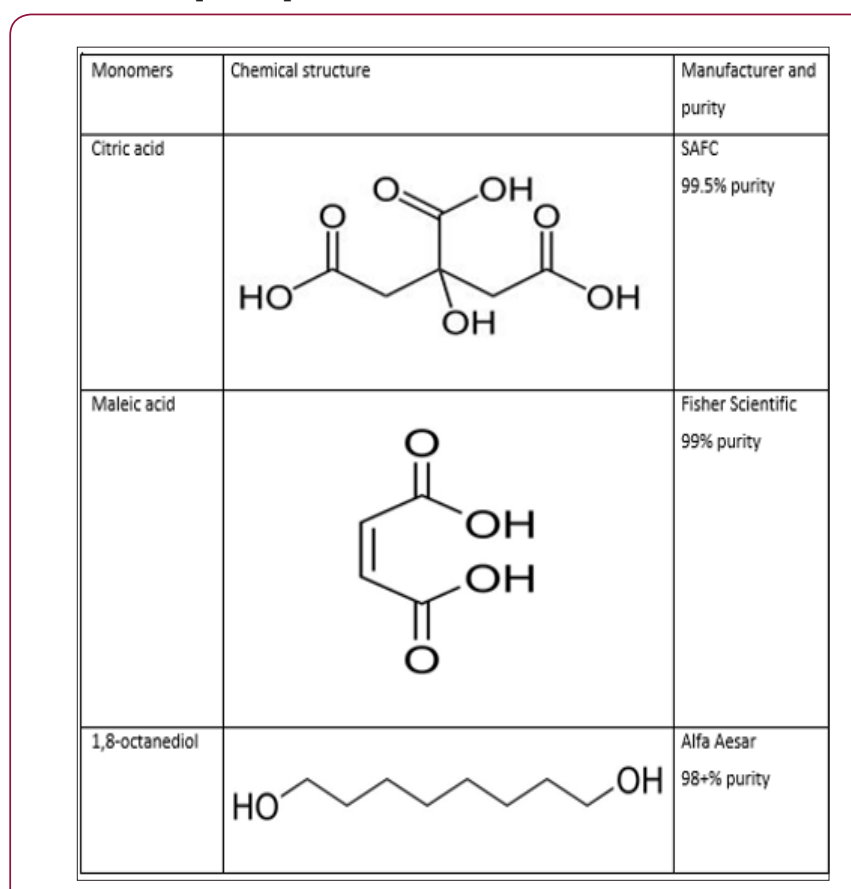

Figure 1: Chemical structure of citric acid, maleic acid and 1,8-octanediol. 


\section{Cross-Linking and Analysis of the Pre-POMC}

Pre-POMC was cross-linked via free radical polymerisation. The freeze-dried pre-POMC was dissolved in DMSO to obtain a polymer solution $(50 \% \mathrm{w} / \mathrm{w}$. UV light was used to crosslink the disks that had Irgacure 2959 and Camphor quinone photo initiator. The POMC specimens were analysed using Differential Scanning Calorimetry (DSC) (Jade machine, serial number 520A8101306) to determine the glass transition temperature ( $\mathrm{Tg}$ ). The sample was heated to $230 \mathrm{oC}$ at a heating rate of $10 \mathrm{oC} / \mathrm{min}$ under nitrogen purge $(20 \mathrm{ml} /$ $\mathrm{min}$ ) to remove any traces of water in the sample. The sample was then cooled to $-50 \mathrm{oC}$ at a rate of $5 \mathrm{oC} / \mathrm{min}$. The sample was held at $-50 \mathrm{oC}$ for 10 minutes. It was then heated from $-50 \mathrm{oC}$ to $20 \mathrm{oC}$ at a heating rate of $10 \mathrm{oC} / \mathrm{min}$, then cooled from $20 \mathrm{oC}$ to $-50 \mathrm{oC}$ at $5 \mathrm{oC} /$ min. It was then held for 10 minutes at $-50 \mathrm{oC}$. It was then heated from $-50 \mathrm{oC}$ to $50 \mathrm{oC}$ at $10 \mathrm{oC} / \mathrm{min}$. The cycle was repeated twice for each sample. Two repeats were carried out. Tensile properties were determined using an Instron 5569A machine. The POMC discs were cut into in a dog-bone-shape using a scalpel. The dimensions of the sample are $10 \mathrm{~mm} \times 5 \mathrm{~mm} \times 1.5 \mathrm{~mm}$; length $\mathrm{x}$ width $\mathrm{x}$ thickness.

\section{In Vitro Degradation of POMC}

Four POMC samples were suspended in $10 \mathrm{mls}$ of PBS solution to determine the degradation rate of the polymer. The discs were weighed before being placed into the PBS solution to determine initial mass (M0). The suspended samples were maintained at $37 \mathrm{oC}$ by placing them in an incubator. The $\mathrm{pH}$ of the PBS buffer solution was checked every day and was replaced every day for the first week and weekly thereafter. This ensured that the $\mathrm{pH}$ remained at 7.4, thereby eliminating accelerated degradation due to acidic product formation. Each week the samples were removed from the PBS, rinsed with distilled water, freeze dried for twenty-four hours and subsequently weighed to determine the remaining mass (Mt). The sample was then placed back into PBS solution and the process was repeated each week for eight weeks.

\section{Dexamethasone and BSA Release}

A standard curve for both Dexamethasone and BSA was established. $1 \mathrm{ml}$ of PBS was measured out into a small glass vial. 6mgs of Dexamethasone was added to this to create a 6mg Dex/ ml PBS solution. This solution was then placed into a cuvette using a pipette, which was then placed into the spectrophotometer. The absorbance of the solution was measured using the spectrophotometer. The solution was then removed from the cuvette using a pipette and placed back into the vial. $1 \mathrm{ml}$ of PBS was added to this to create a $3 \mathrm{mg}$ Dex/ml PBS solution. This was then placed into a cuvette and the absorbance of the solution was measured using the spectrophotometer. $1 \mathrm{ml}$ of PBS was measured out into a new vial. $4 \mathrm{mgs}$ of Dexamethasone was added to this to create a $4 \mathrm{mg}$ Dex/ ml PBS. $1 \mathrm{ml}$ of PBS was added to this to create a $2 \mathrm{mg}$ Dex/ml PBS solution. $1 \mathrm{ml}$ of PBS was added to it to create a $1 \mathrm{mg}$ Dex/ ml PBS solution. The absorbance of the solution was measured using the spectrophotometer [21-39].

\section{Drug Incorporation and Release}

Once a standard curve was determined the drug was added to the pre-POMC solution. $5 \mathrm{mg}$ of Dexamethasone was chosen as it was near the upper limit. This was then poured out to form a disk and left to photo-polymerised under the UV light for 30 minutes. A spectrophotometer was used to determine the release of the BSA and Dexamethasone from POMC. To determine drug, release the samples were placed into a vial containing $1 \mathrm{ml}$ of PBS and incubated at $37 \mathrm{oC}$. At determined intervals the $1 \mathrm{ml}$ of PBS was removed from the vials, using a pipette and placed into a cuvette. The cuvette was then placed into the spectrophotometer to determine absorbance.

\section{Results}

\section{Synthesis and Crosslinking of Pre-POMC}

It was determined that the optimum conditions for pre-POMC synthesis is $130 \mathrm{oC}$ for 60 minutes then $110 \mathrm{oC}$ for 120 minutes under a vacuum and constant nitrogen flow. Both 6:4 pre-POMC and 8:2 pre- POMC were successfully synthesised via a poly-condensation reaction. 6:4 pre-POMC was much more viscous compared to 8:2 pre-POMC. 6:4 POMC was successfully crosslinked using UV and blue light, using Irgacure and Camphorquinone photoinitiators respectively and successfully solidified to form POMC disks. UV light produced a more solidified POMC disk.

\section{Structural Characterisation of Pre-POMC and POMC}

The purified pre-POMC and POMC disks were characterised using FTIR. The spectra confirm that pre-POMC was successfully synthesised with incorporation of all the monomers. The peaks on the spectra are characteristic of specific functional groups within the monomers (Figure 3). The differences between the pre-POMC and POMC FTIR spectra confirm that the crosslinking reaction has occurred successfully. This can be seen in the reduction in the size of the peak at $1642 \mathrm{~cm}^{-1}$. The reduction is a result of the $\mathrm{C}=\mathrm{C}$ bonds from the maleic acid being broken during the free radical polymerisation reaction (Figures 4 \& 5).

\begin{tabular}{|l|l|}
\hline Peak $\left(\mathrm{cm}^{-1}\right)$ & Functional group \\
\hline $1690-1750$ & $\begin{array}{l}\text { Carbonyl groups (C=0) from ester bond } \\
\text { Carboxylic acid from citric acid }\end{array}$ \\
\hline 1642 & Olefin moiety from maleic acid \\
\hline 2931 & Methylene group from 1,8 Octandiol \\
\hline 3490 & Hydroxyl group \\
\hline
\end{tabular}

Figure 3: A Peaks present in FTIR spectra of 6:4 pre-POMC and crosslinked 6:4 POMC. The peaks represent the functional groups present in the polymer. 


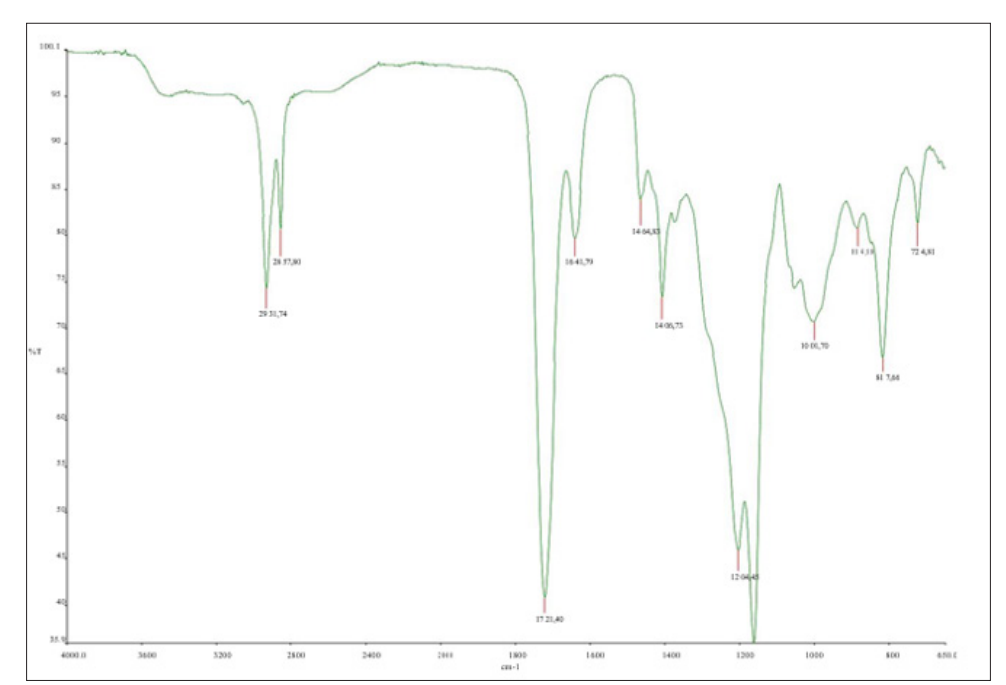

Figure 4: FTIR spectra of pilot 6:4 POMC.

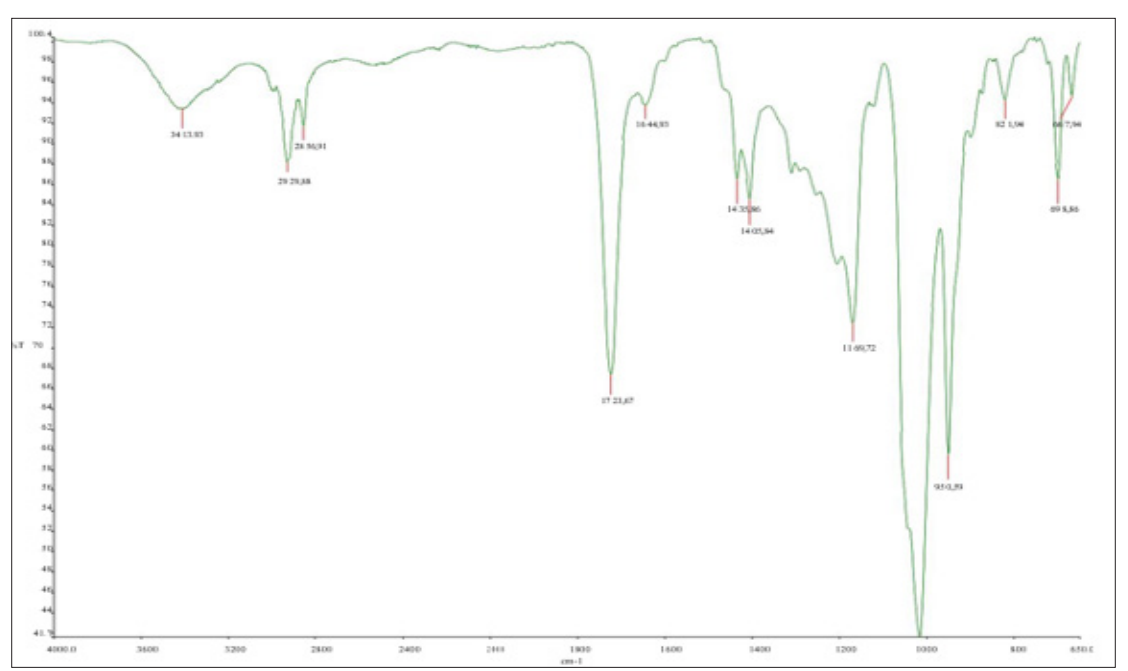

Figure 5: FTIR spectra of 6:4 POMC crosslinked.

\section{Thermal Analysis}

Thermographs for POMC disks were obtained from DSC analysis (Figures 6 \& 7). Pyris software was used to calculate the glass transition using the mid-method point. These results show that the glass transition temperature of the crosslinked POMC is below $0 \mathrm{oC}$, indicating that POMC is completely amorphous at body temperature.

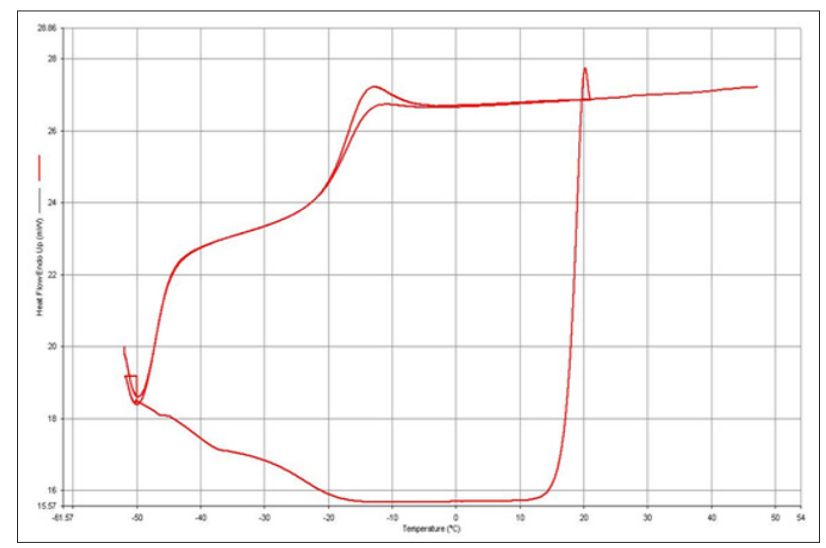

Figure 6: Representative heating and cooling curve cycles for 6:4 POMC disk crosslinked with Irgacure 2959 photo-initiator use UV light. 


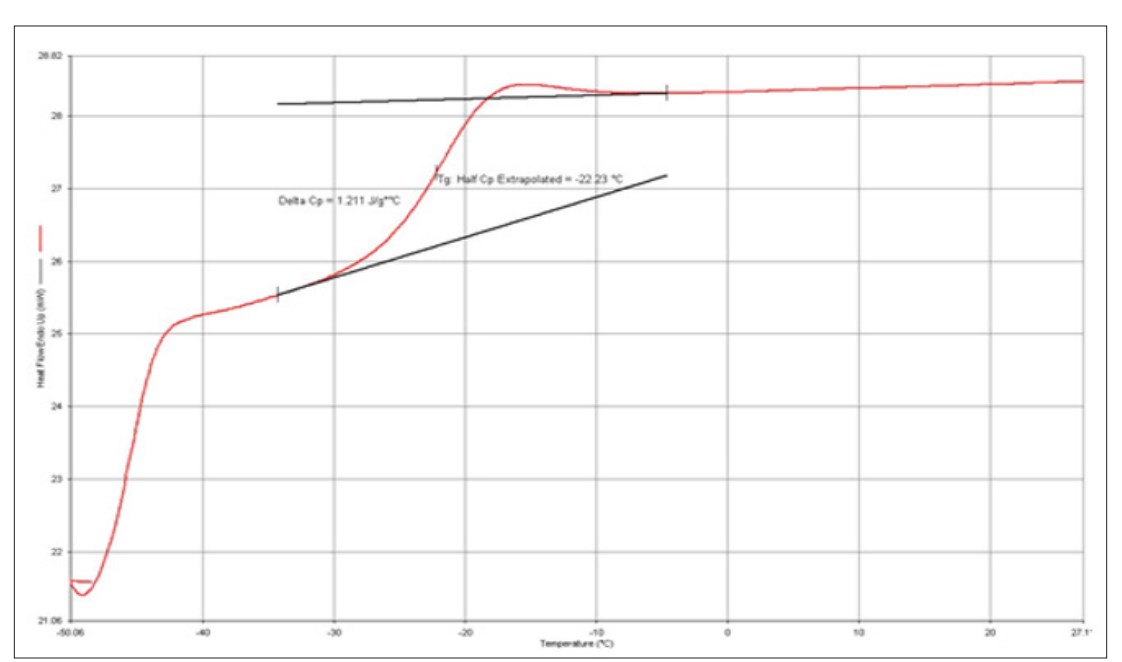

Figure 7: The mid-point method was used to determine the glass transition temperature of the crosslinke 6:4POMC. The second heating curve was used to obtain the glass transition temperature.

\section{Tensile Testing}

Tensile testing found that the maximum load was $1.40 \mathrm{~N}$ Maximum extension was $3.65 \mathrm{~mm}$ and Young's modulus $=0.45 \mathrm{MPa}$
(Figure 8). The results show that the mechanical properties of 6:4 POMC are within the range of the soft tissues in the body (Figures 9-12).

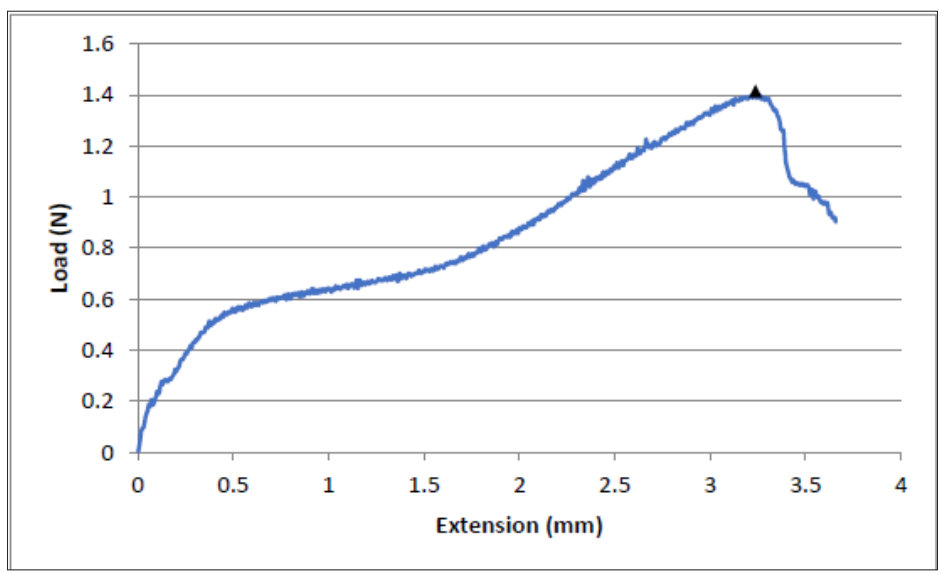

Figure 8: Illustrates the extension (mm) of 6:4 POMC specimen as load $(\mathrm{N})$ is applied.

\begin{tabular}{|c|c|c|c|}
\hline Time(hrs) & Mean Release (MB) & \% Release & Standard Deviation (96) \\
\hline 24 & 1.73 & 34.64 & 1.73 \\
\hline 48 & 1.94 & 38.96 & 4.37 \\
\hline 72 & 2.25 & 45.61 & 5.75 \\
\hline 96 & 2.50 & 50.11 & 5.60 \\
\hline 120 & 2.80 & 55.55 & 5.08 \\
\hline 144 & 3.20 & 64.02 & 4.10 \\
\hline 168 & 3.42 & 68.35 & 2.25 \\
\hline 192 & 3.75 & 75.07 & 3.49 \\
\hline 288 & 3.94 & 78.76 & 4.47 \\
\hline 366 & 3.95 & 79.09 & 4.50 \\
\hline
\end{tabular}

Figure 9: Mean BSA release (Mg and \%) and standard deviation over a 366hr period in PBS solution. 


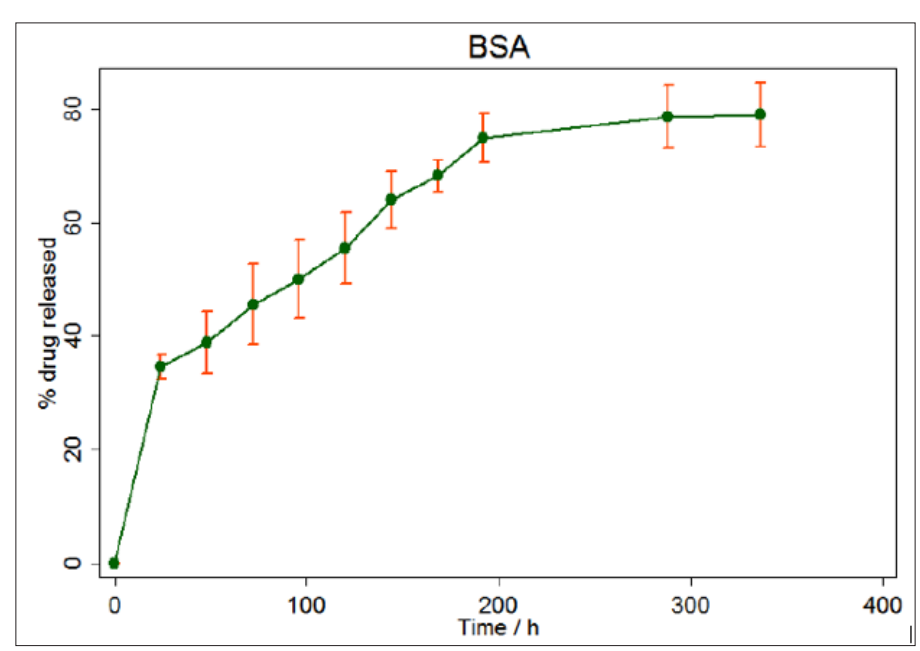

Figure 10: Mean Percentage release of BSA in PBS solution over 366hrs from 6:4 POMC disks crosslinked with Irgacure photoinitiator using UV light.

\begin{tabular}{|cccc|}
\hline Time (Hrs) & $\begin{array}{c}\text { Mean Dexamethasone } \\
\text { Release }(\mathrm{mg})\end{array}$ & $\begin{array}{c}\text { \% Dexamethasone } \\
\text { release }\end{array}$ & Standard Deviation \\
\hline 24 & 2.33 & 46.73 & 3.69 \\
\hline 48 & 2.89 & 56.81 & 5.60 \\
\hline 120 & 3.43 & 68.63 & 4.38 \\
\hline 144 & 3.75 & 75.10 & 7.01 \\
\hline 168 & 4.25 & 85.01 & 4.63 \\
\hline 192 & 4.42 & 88.38 & 3.80 \\
\hline 288 & 4.51 & 90.26 & 2.56 \\
\hline 312 & 4.56 & 91.26 & 2.03 \\
\hline 336 & 4.58 & 91.71 & 2.07 \\
\hline
\end{tabular}

Figure 11: Mean Dexamethasone release (Mg and \%) and standard deviation over a 366hr period.

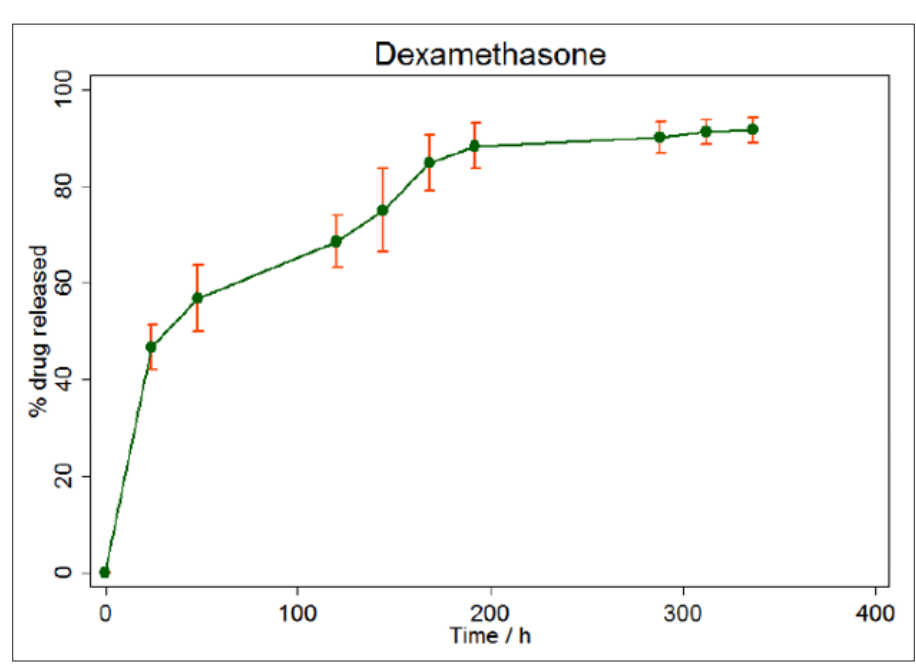

Figure 12: Mean \% Percentage release of Dexamethasone over 366hrs in PBS solution from 6:4 POMC disks crosslinked with Irgacure photo-initiator using UV light. 


\section{Discussion}

The synthesis of POMC was based on the methodology outlined by Gyawali et al. [6], where a low molecular weight pre-polymer (pre-POMC) is synthesised and then crosslinked via photopolymerisation to form a higher molecular weight polymer (POMC). This method gives several advantages over other methods where a high molecular weight polymer is formed in one synthesis reaction. Firstly, the low molecular weight polymer has a low viscosity and can therefore be easily injected into any desired site, giving it the potential for in situ use. It is also able to conform to any shape. Compared to other synthesis methods, the pre-POMC synthesis is much easier, quicker and cheaper.

The photo-polymerisation reaction was successfully carried out at room temperature $(22 \mathrm{oC})$, using UV light and blue light. It is important that the reaction is carried out at low temperatures because at high temperatures any drugs or proteins incorporated into the pre-POMC would become denatured and inactivated. The low crosslinking temperature also indicates that the reaction could be carried out in situ without causing damage to the surrounding tissue.

$1 \%(\mathrm{w} / \mathrm{w})$ photo initiator was used as previous studies Gyawali et al. [6] had determined that this was the optimum amount of photo initiator. If more is used, free radical polymerisation is terminated by the formation of short polymer chains due to the excess of radicals. This reduces the stiffness of the polymer as fewer crosslinks are formed. Jansen et al. [26] recently investigated delivery of cyanobalamin (Vitamin B12) using photo-polymerisation. Similarly, they used 1\% (w/w) Irgacure 2959 photoinitiator. The results showed that there were no detrimental effects to the NIH 3T3 fibroblasts that were used to determine the activity of the drug released following exposure to the photo-initiator or UV light.

One of the main differences between this study and those reported in the literature is the time taken for the pre-POMC disks to photo-polymerise under the UV light. Improving the purity of the materials and freeze drying the pre-POMC multiple times helped to reduce the time it took for the disks to photo-polymerise to less than 60 minutes. 60 minutes in not an acceptable polymerisation time if this system is to be considered for clinical practice.

This study has demonstrated the successful application of photo-polymerisation to create a drug and protein delivery system. The use of mild photo-polymerisation techniques gives the option being able to form this system in situ. In situ polymerisation offers several advantages over other systems. One of the main advantages it that it is a much less invasive technique of drug delivery compared to some of the techniques reported in the literature. For example, PGLA microsphere delivery requires a surgical incision (Jain [11]). By using in situ polymerisation the drug can be introduced to the site via a relatively small needle and photo-polymerisation can be carried out via fibre optic cables utilising techniques similar to those used in arthroscopic procedure (Anseth, 2002).

One important factor that should be considered is the free radical polymerisation technique used for crosslinking. Although it provides an effective and efficient method of crosslinking, it requires the use of photo-initiators that dissociate into free radicals when light is absorbed. The generation of these free radicals could potentially be harmful to surrounding tissues and could cause damage to cellular membranes (Bryant et al., 2000). In this study two different photo-initiators were used; Irgacure 2959 and Camphorquinone. Irgacure 2959 was chosen over Camphorquinone because Camphorquinone required the addition of $\mathrm{N}, \mathrm{N}$ - dimethylp-toluidine initiator, which is a highly toxic substance and could potentially induce tumour promotion. However, this is unlikely as only a very small amount of the initiator was used during the crosslinking reaction.

The FTIR spectra confirm that the synthesis of pre-POMC has been successful as all of the characteristic bonds are present. The peak around $2900 \mathrm{~cm}-1$ represents the methylene group from the 1,8 Octandiol indicating that the 1,8 Octandiol has been successfully incorporated into the pre-POMC. The peak around $1650 \mathrm{~cm}-1$ represents the double bond in the maleic acid group. This indicates that the maleic has been successfully incorporated into the pre-POMC. The peaks around $1720 \mathrm{~cm}-1$ and $1650 \mathrm{~cm}-$ 1suggest the presence of the carbonyl group from the ester bond and carboxylic acid. This indicates that citric acid has been successfully incorporated into the pre-POMC.

After photo-polymerisation the peak around $1650 \mathrm{~cm}-1$ (Maleic acid group) decreased in size. This is because the double bonds in the maleic acid are broken during the crosslinking reaction, indicating that this reaction had been successful.

Tensile strength testing was carried out on crosslinked POMC films. The results indicate that POMC is soft and elastic in nature with a mean initial modulus of $0.35 \mathrm{Mpa}$. The young's modulus is an important property. If POMC is to be used successfully within the body it must have similar mechanical properties as the soft tissues. The mean modulus for POMC obtained in this study is similar to the modulus of some of the soft tissues within the body. For example, the stratum corneum of human skin (0.24-0.40Mpa) and human bladder (0.25Mpa) (Gyawali et al. [6].

The release profiles show that POMC is able to deliver a sustained and controlled release of both BSA and Dexamethasone and statistical analysis shows that there is little standard deviation between the five repeats. The drug release profiles suggest that POMC can provide a sustained release of Dexamethasone and BSA. Although there is an initial burst release of the drug/protein, this only lasts over a 24 hour period and, on average, $34 \%$ of the drug is released over this time. Overall the drug release profile shows a sustained release over a 14 day period, with $84 \%$ of the drug being released on average. Although degradation of the POMC disk contributes to the release of Dexamethasone and BSA, degradation of POMC is unable to account for all of the drug released. This is evident when drug release is compared to mass loss of the POMC disks at specific time points. For example, at 7 days $85 \%$ of the Dexamethasone has been released and $68 \%$ of the BSA has been released, but POMC disks have only lost, on average, $16 \%$ of their mass. This suggests that diffusion is also contributing to the drug 
and protein release. If the release was controlled by diffusion alone it would have resulted in a much quicker release of the drug, over a shorter period of time. If the release was controlled by degradation alone the drug would have been released over a longer period of time. At 14 days the mass loss of the POMC disks is only 25\%, therefore it cannot account for sustained drug release alone. Thus, it is reasonable to conclude that both controlled molecular diffusion and degradation contribute to sustained release of Dexamethasone and BSA from the POMC disks.

The influence of diffusion on drug release is supported by the difference in the release rates of Dexamethasone and BSA; Dexamethasone is released quicker than BSA. This can be attributed to Dexamethasone having a molecular weight of 392Da, much smaller compared the molecular weight of BSA, which is $66,000 \mathrm{Da}$. If diffusion alone was responsible for drug release there would be a larger difference between Dexamethasone and BSA release profiles, as they have very different molecular weights.

Degradation was observed over an 8 week period. The POMC disks suspended in PBS degrade at a significantly faster rate than the disks suspended in distilled water. This difference can again be explained in terms of the ionisation of the polymer chains. In PBS ionised chains result in greater swelling of POMC disk. Swelling is an important process because as more fluid is absorbed, the greater the chances of the ester bond being degraded by hydrolysis.

Degradability is an important property. It removes the need for surgical retrieval of the device after drug/protein has been depleted. It also contributes to the drug and protein release; the faster the POMC is degraded, the quicker the drug/protein will be released.

\section{Conclusion}

This study demonstrates that pre-POMC can be successfully synthesised and crosslinked to form POMC using both UV and blue light systems. Furthermore, this system can be used to successfully incorporate biologically active molecules into the polymer and has demonstrated successful sustained release. The results of this study suggest that this is a very versatile system that can potentially be successfully used in a variety of different applications. In addition, the results demonstrate that POMC can be successfully crosslinked via photopolymerisation, using both UV and blue light systems. This offers an alternative to the harsh processing conditions that limit the application of some of the current biodegradable polymers available. This system could therefore potentially be used for the inclusion of biologically active molecules.

\section{References}

1. Shi R, Chen D, Liu Q, Wu Y, Xu X, et al. (2009) Recent advances in synthetic bioelastomers. International journal of molecular sciences 10(10): 4222-4256.

2. Yang J, Webb A, Hageman G, Ameer G (2004) Novel citric acid-based biodegradable elastomers for tissue engineering. Adv Mater 16(6): 511516.

3. Sodian R, Sperling JS, Martin DP, Egozy A, Stock U, et al. (2000) Fabrication of a trileaflet heart valve scaffold from a polyhydroxyalkanoate biopolyester for use in tissue engineering. Tissue Eng 6(2): 183-188.
4. Gunatillake P, Adhikari P (2003) Biodegradable synthetic polymers for tissue engineering. Eur Cell Mater 5: 1-16.

5. Tran R, Zhang Y, Gyawali D, Yang J (2009) Recent development on citric acid derived biodegradable elastomers. Recent Pat Biomed Eng 2: 216227.

6. Gyawali, D, Nair, P, Zhang Y, Tran R, Zhang C, et al. (2010) Citric acidderived in situ cross-linkable biodegradable polymers for cell delivery. Biomaterials 31(34): 9092-9105.

7. Gyawali D, Tran R, Guleserian K, Tang L, Yang J (2010) Citric acid derived photo-cross-linked biodegradable elastomers. Journal of Biomaterial Scienc 21(13): 1761-1782.

8. Serrano M, Chung E, Ameer G (2010) Advances in applications of biodegradable elastomers in regenerative medicine. Advanced functional materials 20(2): 192-208.

9. Hatefi A, Amsden B (2002) Biodegradable injectable in situ forming drug delivery systems. J Control Release 80(1-3): 9-28.

10. Madan M, Bajaj A, Lewis S, Udupa N (2009) In Situ Forming Polymeric Drug Delivery Systems. Indian J Pharm Sci 71(3): 242-251.

11. Jain $R$ (2000) The manufacturing techniques of various drug loaded biodegradable poly(lactide-co-glycolide) (PGLA) devices. Biomaterials 21(3): 2475-2490.

12. Motlagh D, Allen J, Hoshi R, Yang J, Lui K, et al. (2006) Hemocompatibility evaluation of poly (diol citrate) in vitro for vascular tissue engineering. Wiley Interscience pp. 908-915.

13. Anthasiou K, Agarwal C, Barber F, Burkhart S (1998) Orthpaedic applications for PLA-PGA biodegradable polymers. Arthrosc: J Arthrosc Relt Surg 14(7): 726-737.

14. Ashammakhi N, Rokkanen P (1997) Absorbable polyglycolide devices in trauma and bone surgery. Biomaterials 18(1): 3-9.

15. Bae SE, Choi DH, Han DK, Park K (2010) Effect of temporally controlled release of dexamethasone on in vivo chondrogenic differentiation of mesenchymal stromal cells. J Controlled Release 143(1): 23-30.

16. Bettinger J (2011) Biodegradable Elastomers for Tissue Engineering and Cell-Biomaterial Interactions. Macromolecular Bioscience 11(4): 467-482.

17. Bryant SJ, Anseth KS (2001) The effects of scaffold thickness on tissue engineered cartilage in photocrosslinked poly (ethylene oxide) hydrogels. Biomaterials 22(6): 619-626.

18. Chitakara D, Shikanov A, Kumar N, Domb AJ (2006) Biodegradable injectable in situ depot forming drug delivery systems. Macromol BioSci 6(12): 997-990.

19. Dey J, Xu H, Shen J, Thevenot P, Gondi S, et al. (2008) Development of biodegradable cross-linked urethane-doped polyester elastomers. Biomaterials 29(35): 4637-4649.

20. Djordjevic I, Choudhury N, Dutta N, Kumar S (2009) Synthesis and characterization of novel citric acid based polyester elastomers. Polymer 50(7): 1682-1691.

21. Dunn R, English J, Cowsar D, Vanderbelt D (2004) Biodegradable in-situ forming implants and methods of producing the same, US Pat. 5278201.

22. Gerecht S, Townsend S, Pressler H, Zhu H, Nijst C, et al. (2007) A porous photocurable elastomer for cell encapsulation and culture. Biomaterials 28(32): 4826-4835.

23. Hans ML, Lowman AM (2002) Biodegradable nanoparticles for drug delivery and targeting. Current Opinion in Solid State and Materials Science 6(4): 319-327.

24. Hoshi R, Behl S, Ameer G (2009) Nanoporous biodegradable elastomers. Advanced Materials 21(2): 188-192.

25. Hubbell J, Pathak C, Sawhney A, Desai N, Hill J (1995) Photopolymerizable 
biodegradable hydrogels as tissue contacting materials and controlled release carriers, US Pat. $5410016,25$.

26. Jansen J, Boerakker MJ, Heuts J, Feijen J, Grijpma DW (2010) Rapid photo-crosslinking of fumaric acid monoethyl ester-functionalized poly (trimethylene carbonate) oligomers for drug delivery applications. J Controlled Release 147(1): 54-61.

27. Kibbe M, Martinez J, Popowich D, Kapadia M, Ahanchi S, et al. (2010) Citric acid-based elastomers provide a biocompatible interface for vascular grafts. Journal of Biomedical Materials Research Part A 93(1): 314-324.

28. Mahdavi A, Ferreira L, Sundback C, Nichol J, Chan E, et al. (2008) A biodegradable and biocompatible gecko-inspired tissue adhesive. Proceedings of the National Academy of Sciences 105(7): 2307-2312.

29. Middleton J, Tipton A (2000) Synthetic biodegradable polymers as orthopedic devices. Biomaterials 21(23): 2335-2346.

30. Nijst C, Bruggeman J, Karp J, Ferreira L, Zumbuehl, et al. (2007) Synthesis and characterization of photo-curable elastomers from poly(glycerolco-sebacate). Biomacromolecules 8(10): 3067-3073.

31. Nivasu V, Reddy T, Tammishetti S (2004) In situ polymerizable polyethyleneglycol containing polyesterpolyol acrylates for tissue sealant applications. Biomaterials 25(16): 3283-3291.

32. Qing Y, Wenying X, Rånby B (1991) Photoinitiated crosslinking of lowdensity polyethylene I: Reaction and kinetics. Polymer Engineering \& Science 31(22): 1561-1566.

ISSN: 2574-1241

DOI: 10.26717/BJSTR.2019.13.002441

Kiran Dhaliwal. Biomed J Sci \& Tech Res

(C) (P) This work is licensed under Creative

Submission Link: https://biomedres.us/submit-manuscript.php
33. Qiu H, Yang J, Kodali P, Koh J, Ameer GA (2006) A citric acid-based hydroxyapatite composite for orthopedic implants. Biomaterials 27(34): 5845-5854.

34. Sun Z, Chen C, Sun M, Ai C, Lu X, et al. (2009) The application of poly (glycerol-sebacate) as biodegradable drug carrier. Biomaterials 30(28): 5209-5214.

35. Tanihara M, Suzuki Y, NishimuraY, Suzuki K, Kakimaru Y, et al. (1999) A novel microbial infection-responsive drug release system. Journal of Pharmaceutical Sciences, 88(5): 510-514.

36. Taylor M, Daniels A, Andriano K, Heller J (1994) Six bioabsorbale polymers: In-vitro acute toxicity of accumulated degradation products. J Appl Biomater 5(2): 151-157.

37. Tran R, Thevenot T, Gyawali D, Chiao J, Tang L, et al. (2010) Development of a novel biodegradable elastomer featuring a dual crosslinking mechanism for soft tissue engineering. Soft Matter 6(11): 2449-2461.

38. Wang Y, Ameer A, Sheppard B, Langer R (2002) A tough biodegradable elastomer. Nat Biotechnol 20(6): 602-606.

39. Webb R, Yang J, Ameer A (2008) A new strategy to characterize the extent of reaction of thermoset elastomers. Journal of Polymer Science Part A: Polymer Chemistry 46(4): 1318-1328.

\begin{tabular}{ll} 
BIOMEDICAL & Assets of Publishing with us \\
RESEARCHES & - Global archiving of articles \\
\hline ISSN: $2574-1241$ & - Immediate, unrestricted online access \\
\end{tabular}

